\title{
Before and After: Can we predict and/or understand an observed twin law using extant tools and a look at plausible composition planes?
}

\author{
Bruce M. Foxman a and Victor G. Young, Jr. ${ }^{\mathrm{b}}$ \\ aDepartment of Chemistry, MS015, Brandeis University, Waltham, MA 02453-2700; 'Department of \\ Chemistry, The University of Minnesota, Minneapolis, MN 55455
}

The analysis of phase transitions, along with the twinning that may occur during the phase transition has prompted us to further develop our knowledge of twin laws, as well as the relationship between obliquity and the actual twins commonly observed in molecular crystals. For this we needed a copy of Yvon Le Page's program OBLIQUE, ${ }^{1}$ for which the code and executables are now unavailable. We were fortunate enough to have some stimulating discussions with the late Professor Howard Flack, who provided a copy of CREDUC81, an advanced version of Le Page's 1982 program. ${ }^{2}$ Studying the original CREDUC81 program led us to write our own code, which provides an improved analysis of the twin laws suggested by OBLIQUE, including twin laws shown to have been missed ${ }^{3,4}$ by OBLIQUE. The new code is a highly modified version of CREDUC81 that reports all possible twin laws, including obliquity and the twin law matrices. The program, OMEGA v2.10, is available at the Brandeis website. ${ }^{5}$ Our current approach involves (a) use of OMEGA to obtain a list of twin laws, obliquities and matrices followed by (b) inspection of the composition planes for the structure under consideration. "Before" the structure analysis has begun, the results from OMEGA can guide us to a likely twin law, and/or that analysis can be compared, e.g., to a CELL_NOW run or other observation. "After" solution of the structure, the analysis includes a view of the likely choice for the composition plane as well. The presentation will include worked examples, including those where the composition plane may readily be "guessed", along with more complicated cases. Under favorable circumstances, such an approach can work extremely well, and can provide much new information about the chemical nature of the composition plane. Time and progress permitting, we will show examples of the likely layer groups for example composition planes.

1. Le Page, Y. J. Appl. Cryst. 2002, 35, 175-181.

2. Le Page, Y. J. Appl. Cryst. 1982, 15, 255-259.

3. Grimmer, H. Acta Crystallogr., Sect. A 2003, A59, 287-296.

4. Grimmer, H.; Kunze, K. Acta Crystallogr., Sect. A 2004, A60, 220-232.

5. http://people.brandeis.edu/\%7Efoxman1/teaching/omega2.zip. 